\title{
SIGNALS OF PRIMORDIAL PHASE TRANSITIONS ON CMB MAPS
}

\author{
P.S. CORASANITI ${ }^{1,2}$, L. AMENDOLA ${ }^{2}$, F. OCCHIONERO ${ }^{2}$ \\ ${ }^{1}$ Centre for Theoretical Physics, University of Sussex, Falmer, Brighton BN1 9QJ, \\ United Kingdom \\ ${ }^{2}$ Osservatorio Astronomico di Roma, Viale del Parco Mellini 84, 00136 Roma, \\ Italy
}

\begin{abstract}
The analysis of the CMB anisotropies is a rich source of cosmological informations. In our study, we simulated the signals produced by the relics of a first order phase transition occured during an inflationary epoch in the early Universe. These relics are bubbles of true vacuum that leave a characteristic non-Gaussian imprint on the CMB. We use different statistical estimators in order to evaluate this nonGaussianity. We obtain some limits on the allowed values of the bubble parameters comparing our results with the experimental data. We also predict the possibility to detect this signal with the next high resolution experiments.
\end{abstract}

\section{Introduction}

The models of structure formation assume the existence of primordial matter density perturbations, that at the decoupling are source of the temperature fluctuations in the Cosmic Microwave Background (CMB). Currently we have two sets of theories that can explain the formation of these initial perturbations. In the inflationary models with second order phase transition, the quantum fluctuations of the Inflaton field produce Gaussian and scale invariant density perturbations. On the other hand, in models with a transition of the first order, as topological defects and extended inflation, the perturbations are non-Gaussian and scale dependent. Therefore the statistical properties of the CMB anisotropies are a powerful tool in order to distinguish these models. In our study we analyse the non-Gaussianity produced by the relics of a first order phase transition in the context of a specific realization of the extended inflation, built on a non-minimal generalization of Quadratic Gravity ${ }^{1}$. These relics are bubbles of true vacuum that contribute together with the ordinary quantum perturbations to structure formation. In particular these spherical defects can explain the presence of voids observed in several galaxy surveys ${ }^{2}$. The parameters describing the void distribution are the central density contrast $\delta$ of the bubble, its radius $R$ and the fraction $X$ of the space filled by the voids. At the decoupling the bubbles leave a characteristic imprint on the $\mathrm{CMB}^{3}$. In fact solving the linearized Boltzmann-Einstein equation for a bubble perturbation, intersecting the last scattering surface, we have a signal that appear like a cold spot surrounded by an hot isothermal ring, on the scale of the sound horizon at the decoupling. Therefore the bubble imprint is a source of non-Gaussianity.

\section{Non-Gaussian analysis}

We studied the statistical properties of the bubble anisotropies with two different estimators, the three point collapsed function $C_{3}(\alpha)$ and the normalised bispectrum ${ }^{4}$ 
$I_{l}^{3}$. Both estimate the third order correlation of the CMB anisotropies, the former in the real space and the latter in the multipole space.

The $C_{3}(\alpha)$ has been used to compare the bubble non-Gaussianity with the cosmic variance of low resolution observations (COBE). Using the formalism of Magueijo ${ }^{5}$ for the texture-spot anisotropies we calculated an analytical formula of the 3collapsed function for a possonian bubble distribution on the sky. We considered voids with a present radius of $30 h^{-1} M p c$ and with a filled space fraction $X \sim 0.3,0.5$, in agreement with the properties of the voids observed in the galaxy surveys. With these parameters the three collapsed function has been evaluated for different values of the bubble density contrast. Comparing the results with the COBE 4 YEARS data ${ }^{6}$, we obtain an upper limit on $\delta{ }^{7}$. In particular from a chi-square analysis we have $\delta \leq 0.0017$ and $X \leq 0.54$.

In high resolution experiments the bubble signal should be more evident. In order to detect it with a non-Gaussian analysis we used the $I_{l}^{3}$ bispectrum. This estimator is very efficient and not to depend on the cosmological model, being normalized with an appropriate power of the CMB spectrum. We inferred the probability distribution function of the bispectrum, in the range of multipoles $l \in(150,250)$, by Monte Carlo simulations of full CMB sky maps with a resolution of $10^{\prime}$ arcmin. We have that the distributions obtained by the Gaussian simulations are different from those obtained by bubble ones. In particular the latter have a variance much larger than in the Gaussian case. On the contrary at low multipoles these probability functions become identical, as we expect from the Central Limit Theorem. Therefore, the next high resolution observations from Map and Planck could detect and distinguish the non-Gaussianity produced by primordial voids.

\section{References}

1. F. Occhionero \& L. Amendola, 1994, Phys. Rev D 50, 4846.

2. H. El-Ad, T. Piran \& L.N. da Costa, 1996, Ap. J. Lett. 462, L13.

3. L. Amendola, C. Baccigalupi \& F. Occhionero, 1998, Ap. J. Lett. 492, L5.

4. P. Ferreira, J. Magueijo \& K.M. Gorski, 1998, Ap. J. 503,1.

5. J. Magueijo, 1995, Phys. Rev D 52, 4361.

6. A. Kogut et al., 1996, Ap. J. Lett. 464, L29.

7. P.S. Corasaniti, L. Amendola \& F. Occhionero, 1999, Mont. Not. R. Astron. Soc. in press, astro-ph/0005575. 